\title{
Unsteady MHD Natural Convective Flow over Vertical Plate in Thermally Stratified Media with Variable Viscosity and Thermal Conductivity
}

\author{
D. Iranian \\ Department of Mathematics \\ Panimalar Institute of Technology \\ Chennai, India
}

\author{
P. Loganathan \\ Department of Mathematics \\ College of Engineering, Guindy \\ Chennai, India
}

\author{
P. Ganesan \\ Department of Mathematics \\ College of Engineering, Guindy \\ Chennai, India
}

\begin{abstract}
The effects of exponentially varying viscosity and linearly varying thermal conductivity on unsteady MHD natural convective flow past a semi infinite vertical plate in a thermally stratified medium is studied. The variables of both viscosity and thermal conductivity are considered only a function of temperature. The governing boundary layer equations of continuity, momentum and energy have been transformed into dimensionless coupled and nonlinear equations and after that solved by implicit finite-difference method of Crank-Nicolson type. The effects of the varying viscosity, thermal conductivity and stratification parameter at various times are discussed with velocity and temperature profiles and additionally the skin-friction and the rate of heat transfer.
\end{abstract}

\section{Keywords}

Unsteady, vertical plate, variable viscosity, thermal conductivity, thermal stratification.

\section{NOMENCLATURE}

$u, v \quad$ velocity of the fluid in $\mathrm{x}, \mathrm{y}$ directions respectively

$U, V$ dimensionless velocity of the fluid in $X, Y$ directions respectively

Gr Grashof number

$C_{p} \quad$ specific heat at constant temperature

$k \quad$ thermal conductivity of the fluid

M magnetic parameter

$B_{0} \quad$ magnetic induction

$t^{\prime} \quad$ time

$t \quad$ dimensionless time

$L \quad$ characteristic length of the plate

$T$ temperature of the fluid in the boundary layer

$T_{w} \quad$ plate temperature

$T_{\infty} \quad$ temperature of the fluid far away from the plate

$S \quad$ dimensionless stratified parameter

Pr Prandtl number

$N u_{x} \quad$ dimensionless local heat transfer rate

$\overline{N u}$ dimensionless average Nusselt number

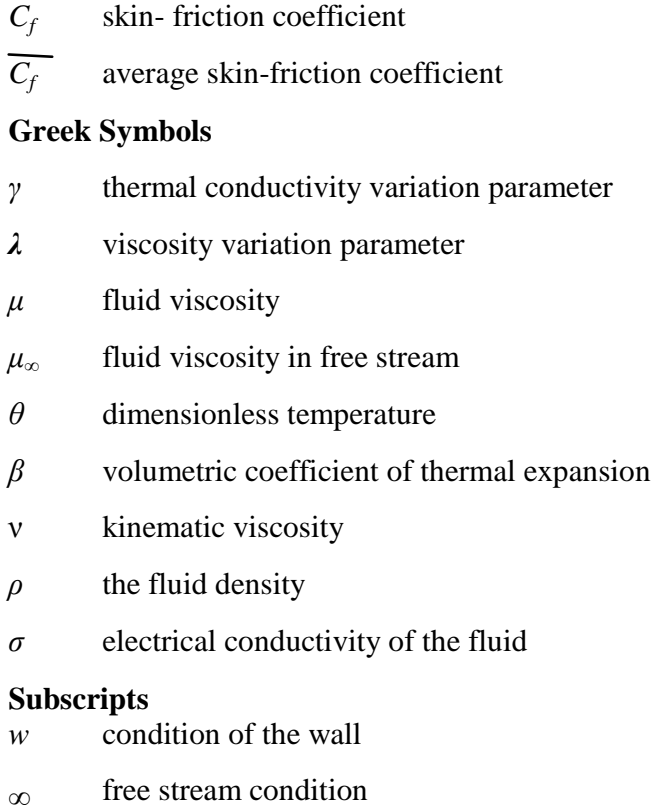

\section{INTRODUCTION}

Free convection flows are of great interest in a number of industrial applications, for example, geothermal frameworks, fiber and granular insulation etc. Buoyancy is also of importance in an environment where differences between land and air temperatures can give rise to complicated flow patterns. Heat transfer on fluid flow due to free convection in the presence of magnetic field finds subsidiary applications in different branches of Science and Technology such as atomic science, fire engineering, computational astrophysics, the magnetic behavior of plasmas in fusion reactors, liquid-metal cooling of nuclear reactors and electromagnetic casting. The problem of free convection flow past an infinite vertical plate under different plate conditions was studied by numerous researchers. Soundalgekar [1] studied the free convection effects on the Stokes problem for an infinite vertical plate by using Laplace transform method. Hellums and Churchill [2] were the first to present unsteady natural convective flow past a semi-infinite isothermal vertical plate by using an explicit finite-difference scheme. As explicit finite-difference scheme has its own particular lacks, later, Soundalgekar and Ganesan [3] have solved free convection flow past a vertical plate with mass transfer using implicit finite difference method of Crank-Nicolson type which is unconditionally stable and convergent. 
Magnetohydrodynamics has attracted the attention of a large number of scholars due to its diverse applications. The study of effects of magnetic field on free convection flow is important in liquid-metals, electrolytes and ionized gases. Soundalgekar and Mohammed Ali [4] have studied free convection effects on MHD flow past an impulsively started infinite vertical isothermal plate. Seddeek [5] reviewed the free convection effects with variable viscosity in an aligned magnetic field. Elbashbeshy [6] studied free convection flow with variable viscosity and thermal diffusivity along a vertical plate in the presence of magnetic field, in which the modified fourth order Runge-Kutta integration scheme was used along with Nachtsheim-Swigert shooting technique. The above problems were not done in the thermally stratified media.

For many fluid flows of practical importance in nature and also in numerous engineering contrivances, the environment is thermally stratified. In the situation of thermal stratification takes place, the fluid temperature and convection exist in such environment like oceans, lakes, solar ponds, nuclear reactors where coolant is present in magnetic field etc. The consideration of researchers on the convective heat transfer in thermal stratified fluid has a consequential topic for scientific enquiry of both theoretical and applied fields. Deka and Neog [7] studied unsteady natural convection flow past an accelerated vertical plate in a thermally stratified fluid. Kulkarni et al. [8] presented the similarity solutions for natural convection flow over an isothermal vertical wall immersed in thermally stratified medium. Gurminder Singh et al. [9] examined the impacts of thermally stratified ambient fluid on MHD convective along a moving non-isothermal vertical plate using Runge-Kutta fourth order method along with shooting technique. The natural convection flow from a continuously moving vertical surface immersed in thermally stratified medium is presented by Takhar et al. [10]. In case of vertical plate problems thermal stratification arises mainly because of temperature variations of different density. The natural convection flow with combined effects due to thermal and mass diffusion in thermally stratified media was studied by Saha and Hossain [11], in which the authors used sixth order implicit Runge-Kutta-Butcher technique.

In many transport processes in nature and in modern applications, the heat transfer with variable viscosity is a consequence of buoyancy effect caused by the diffusion of heat. Hazarika and Sarma [12] concentrated on to get the effects of variable viscosity and thermal conductivity on steady free heat and mass transfer flow along a vertical plate in the presence of a magnetic field. Mahanti and Gaur [13] studied the effects of varying viscosity and thermal conductivity on steady free convective flow and heat transfer along an isothermal vertical plate in the presence of heat sink. Gnaneswara Reddy and Bhaskar Reddy [14] studied unsteady MHD convective heat and mass transfer past a semi-infinite vertical porous plate with variable viscosity and thermal conductivity using the shooting method. Thakur and Hazarika [15] studied the effects of variable viscosity and thermal conductivity on unsteady free convective heat and mass transfer MHD flow of micropolar fluid with constant heat flux through a porous medium.

From all these analysis, it is identified that the variation of viscosity and thermal conductivity with temperature is an interesting area in the stratified fluid flow problems. Most of the above problems dealt with two-dimensional steady MHD boundary layer flow. Hence the focus of the present paper is on unsteady MHD free convective thermally stratified flow with variation of viscosity and thermal conductivity over an isothermal semi-infinite vertical plate.

\section{MATHEMATICAL ANALYSIS}

A two dimensional unsteady flow of a viscous incompressible stratified fluid past a semi-infinite vertical plate is considered. The $\mathrm{x}$ - axis is taken along the plate in the vertically upward direction and the y-axis is chosen normal to the plate as shown in Figure 1. The gravitational acceleration $\mathrm{g}$ is acting downward. Initially, the plate and the fluid are at the same temperature $T_{\infty}$. At time $t>0$, the temperature of the plate is suddenly raised to $T_{w}$ and maintained at the same value. A magnetic field is applied transverse to the direction of the flow. The variable viscosity and thermal conductivity are involved and the viscous dissipation is negligible in the fluid flow. It is assumed that the viscosity of the fluid is an exponential function and that the thermal conductivity is a linear function of the temperature. All the fluid physical properties are assumed to be constant except for the body force terms.

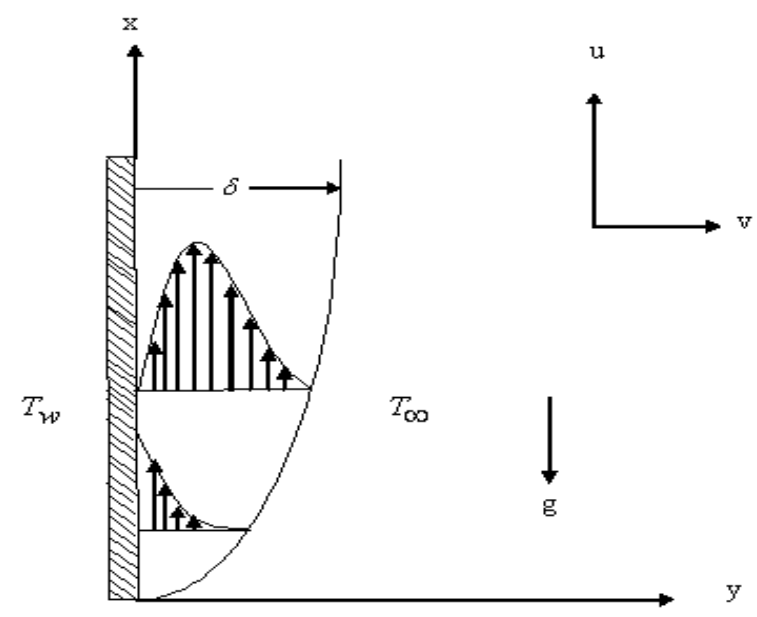

Fig 1: Physical model of the problem

Under these assumptions, the governing boundary layer equations of continuity, momentum and energy with Boussinesq's approximation are as follows

$$
\begin{aligned}
& \frac{\partial u}{\partial x}+\frac{\partial u}{\partial v}=0 \\
& \frac{\partial u}{\partial t}+u \frac{\partial u}{\partial x}+v \frac{\partial u}{\partial y}=\frac{1}{\rho} \frac{\partial}{\partial y}\left(\mu \frac{\partial u}{\partial y}\right)+g \beta\left(T-T_{\infty, x}\right)-\frac{\sigma B_{0}^{2} u}{\rho} \\
& \frac{\partial T}{\partial t}+u \frac{\partial T}{\partial x}+v \frac{\partial T}{\partial y}=\frac{1}{\rho C_{p}} \frac{\partial}{\partial y}\left(k \frac{\partial T}{\partial y}\right)
\end{aligned}
$$

The initial and boundary conditions are

$$
\begin{array}{ll}
t \leq 0, \quad u=0, & v=0, \quad T=T_{\infty, x} \text { for all } x \text { and } y \\
t>0, & u=0, \quad v=0, \quad T=T_{w} \quad \text { at } y=0 \\
& u=0, \quad v=0, \quad T=T_{\infty, 0} \quad \text { at } x=0 \\
& u \rightarrow 0, \quad T \rightarrow T_{\infty, x} \quad \text { at } y \rightarrow \infty
\end{array}
$$


Introducing the following non dimensional quantities

$$
\begin{aligned}
& X=\frac{x}{L}, Y=\frac{y G r^{1 / 4}}{L}, U=\frac{u L G r^{-1 / 2}}{v}, V=\frac{\nu L G r^{-1 / 4}}{v}, M=\frac{\sigma B_{0}^{2} L^{2} G r^{-1 / 2}}{\rho v} \\
& \theta=\frac{T_{w}-T_{\infty, x}}{T_{w}-T_{\infty, 0}}, t^{\prime}=\frac{t v G r^{1 / 2}}{L^{2}}, \operatorname{Pr}=\frac{\nu \rho C_{p}}{k_{\infty}}, G r=\frac{g \beta L^{3}}{v^{2}}\left(T_{w}-T_{\infty, 0}\right)
\end{aligned}
$$

The variations of the normalized viscosity and thermal conductivity parameters are composed in the form (Elbashbeshy and Ibrahim [16], Elbarbary and Elgazery [17]):

$$
\begin{aligned}
& \mu(\theta) / \mu_{\infty}=\exp (-\lambda \theta) \\
& k(\theta) / k_{\infty}=1+\gamma \theta
\end{aligned}
$$

By introducing the above non dimensional quantities and the parameters $(6,7)$ in equations (1), (2) and (3), they are reduced to the non-dimensional form as follows

$$
\begin{aligned}
& \frac{\partial U}{\partial X}+\frac{\partial V}{\partial Y}=0 \\
& \frac{\partial U}{\partial t^{\prime}}+U \frac{\partial U}{\partial X}+V \frac{\partial U}{\partial Y}=\exp (-\lambda \theta)\left(\frac{\partial^{2} U}{\partial Y^{2}}-\lambda \frac{\partial U}{\partial Y} \frac{\partial \theta}{\partial Y}\right)+\theta-M U \\
& \frac{\partial \theta}{\partial t^{\prime}}+U \frac{\partial \theta}{\partial X}+U S+V \frac{\partial \theta}{\partial Y}=\frac{1}{\operatorname{Pr}}\left[(1+\gamma \theta) \frac{\partial^{2} \theta}{\partial Y^{2}}+\gamma\left(\frac{\partial \theta}{\partial Y}\right)^{2}\right]
\end{aligned}
$$

We obtain the boundary condition for temperature at the wall in non-dimensional form as follows

$$
\theta=\frac{T_{w}-T_{\infty, x}}{T_{w}-T_{\infty, 0}}=1-\frac{T_{\infty, x}-T_{\infty, 0}}{T_{w}-T_{\infty, 0}} . \text { Since } T_{\infty, x} \text { is a }
$$

linear function, $\quad \theta=1-\frac{1}{\Delta T_{0}} \frac{d T_{\infty, x}}{d X} X=1-S X, \quad$ where

$$
\Delta T_{0}=T_{w}-T_{\infty, 0} \quad \text { and } \quad S=\frac{1}{\Delta T_{0}} \frac{d T_{\infty, x}}{d X} .
$$

The corresponding initial and boundary conditions of (4) are

$$
\begin{aligned}
t^{\prime} \leq 0: U & =0, V=0, \theta=0 \quad \text { for all } X \text { and } Y \\
t^{\prime}>0: U & =0, V=0, \theta=1-S X \text { at } Y=0 \\
U & =0, V=0, \theta=0 \text { at } X=0 \\
U & \rightarrow 0, \theta \rightarrow 0 \text { as } Y \rightarrow \infty
\end{aligned}
$$

\section{NUMERICAL TECHNIQUE}

The governing equations (8-10) are unsteady, coupled and nonlinear with initial and boundary conditions (11). Using implicit finite-difference scheme of Crank-Nicolson type, the governing equations are solved as described (Thomas algorithm) in Carnahan et. al [18]. The region of integration for the present problem is considered as a rectangle composed of the lines indicating $X_{\max }=1$ and $Y_{\max }=15$, where $Y_{\max }$ corresponds to $\mathrm{Y}=\infty$, which lies well outside both the momentum and energy boundary layers. At a particular time level $\mathrm{n}$, finite difference equations at every internal nodal point on a particular i-level constitutes a tridiagonal system of equations and thus the values of $U$ and $\theta$ are known at every nodal point on a particular i level at $(n+1)^{\text {th }}$ time level and determinately, the values of $\mathrm{V}$ are calculated explicitly at every nodal point on a particular $\mathrm{i}$ level at $(\mathrm{n}+1)^{\text {th }}$ time level. In a similar manner, computations are carried out by moving along i direction. After computing values corresponding to each $i$ at $(n+1)^{\text {th }}$ time level, the values at the next time level are resolute in a similar manner. Computations are completed until the steady-state is reached. The steady-state solution is assumed to have been reached, when the absolute difference between the values of $U$, as well as temperature $\theta$ at two consecutive time steps are less than $10^{-5}$ at all grid points.

The finite difference scheme is proved to be unconditionally stable, for a natural convective flow, using Von-Neumann technique. The local truncation error is $\mathrm{O}\left(\Delta \mathrm{t}^{\prime}{ }^{2}+\Delta \mathrm{Y}^{2}+\Delta \mathrm{X}\right)$ and it tends to zero as $\Delta \mathrm{t}^{\prime}, \Delta \mathrm{Y}$, and $\Delta \mathrm{X}$ tend to zero, which demonstrates that the scheme is compatible. Hence compatibility and stability ensure the implicit finite difference scheme is convergent.

\section{RESULTS AND DISCUSSION}

The computations of dimensionless velocity, temperature profiles have been carried out for different values of the parameters. The velocity and temperature profiles obtained in a dimensionless form are presented in Figures (2-8) for $\mathrm{Pr}=0.73$ (air) and $\operatorname{Pr}=7.0$ (water). Figure 2 represents the velocity profile for different values of $S$ and for the fixed values of $\lambda=-0.6$, $\gamma=1.0$ and $\mathrm{M}=0$. It is clear from the Figure that the time to reach steady state decreases gradually with the increase of thermal stratification parameter S. The velocity increases with time until a temporal maximum $(\mathrm{U}=0.53922)$ is reached and after there is a reduction can be seen until the steady state is reached. Figure 3 depicts the velocity profile for different values of magnetic parameter $\mathrm{M}$. It is observed from the figure that the velocity decreases as $M$ increases with time increasing and reaches temporal maximum and then decrease to reach steady state value. The Figures 4 and 5 represent the velocity profile for Prandtl number $(=7.0)$ for water. The velocity profile is demonstrated in Figure 4 for different values of S. It shows that the velocity decreases as $\mathrm{S}$ increases. The velocity reaches temporal maximum at $(\mathrm{U}=0.92015)$ and decreases monotonically to reach steady state. The steady state velocity can be seen from Figure 5 for different values of thermal conductivity parameter $\gamma$, magnetic parameter $\mathrm{M}$ and for some fixed values of $S=0, \lambda=0.5$. The velocity decreases as decreasing the value of $\gamma$ but increasing the value of $\mathrm{M}$.

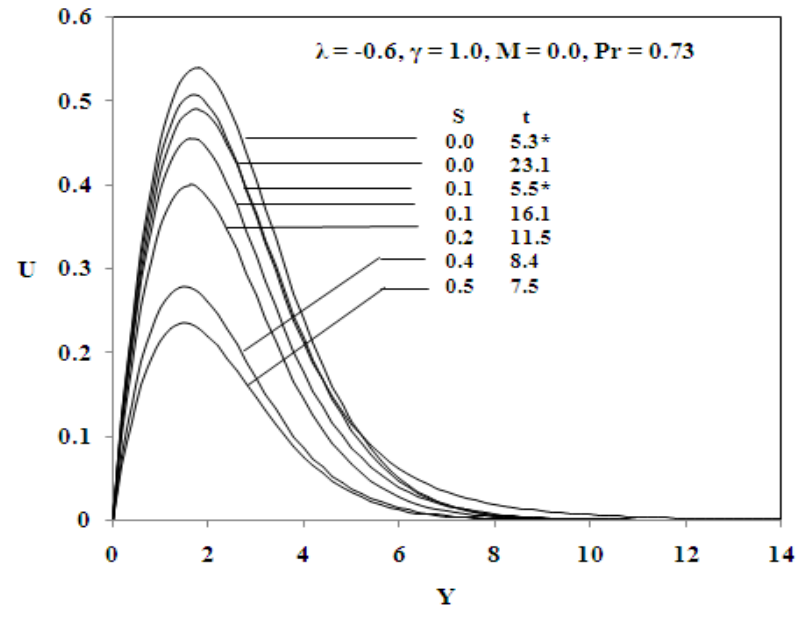

Fig 2: Steady state velocity for different values of $S$ (* Temporal maximum) 


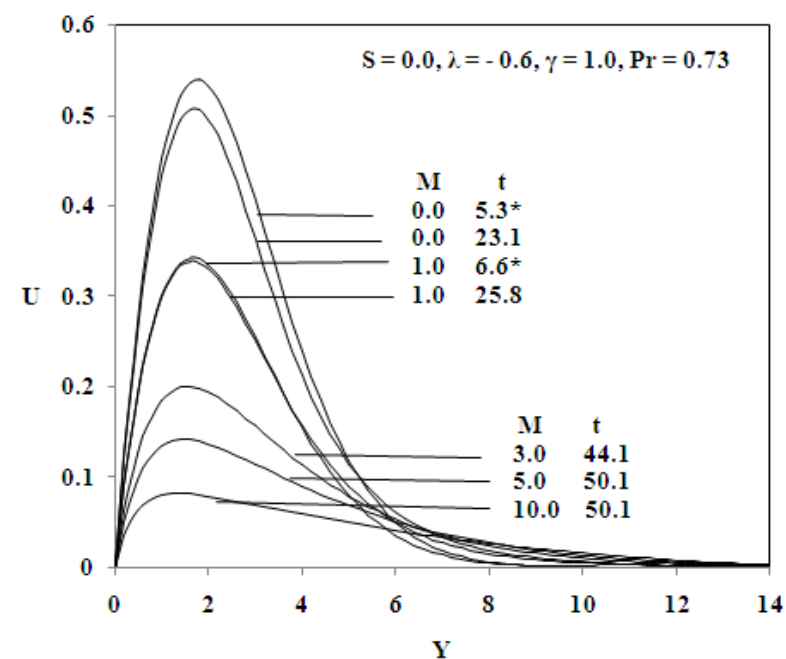

Fig 3: Steady state velocity profiles for different values of $M$ (* Temporal maximum)

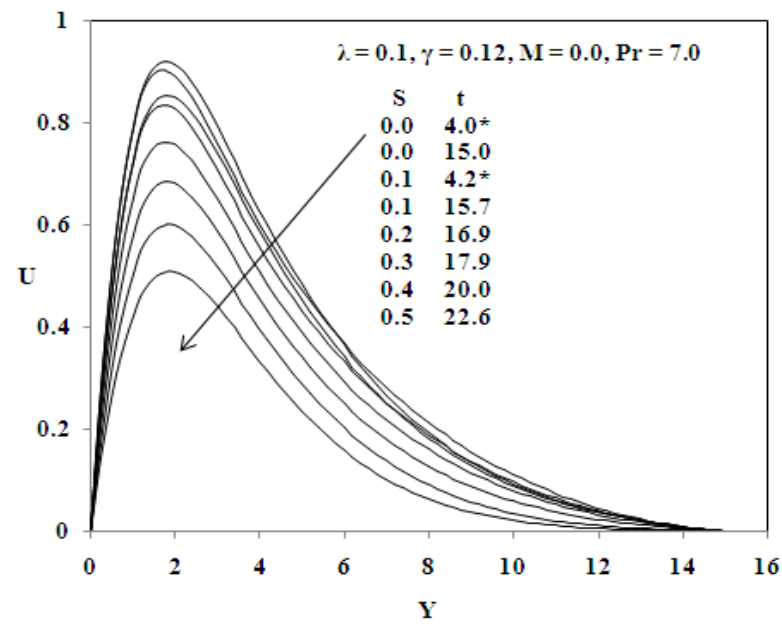

Fig 4: Steady state velocity profiles for different values of $S$ (*Temporal maximum)

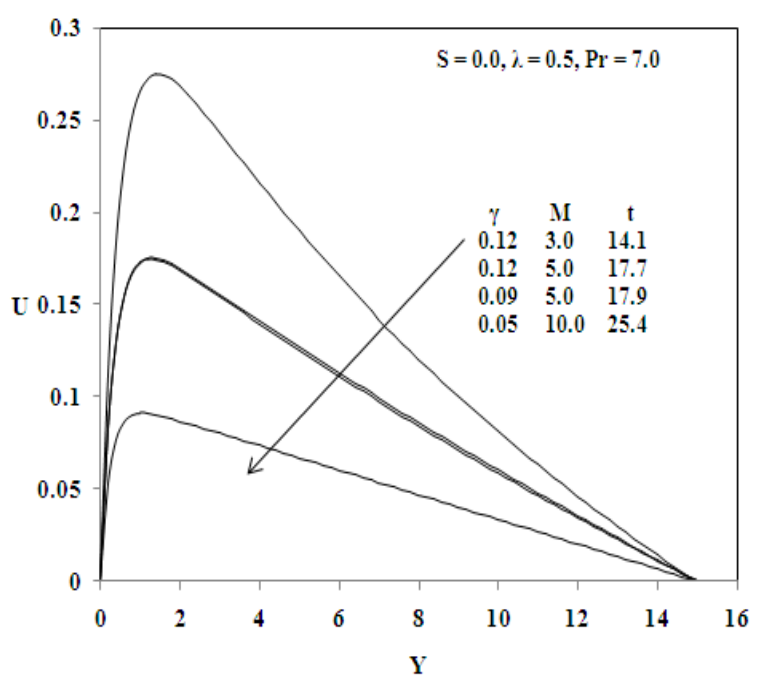

Fig 5: Steady state velocity profiles for different values of $\gamma$ and $M$
The impact of the stratified parameter on the temperature profiles is shown in Figure 6. It shows that the temperature profiles for air. The temperature profile decreases as the stratified parameter increases with decreasing time for some fixed values of $\lambda, \gamma$ and M. It can be seen that the values of the non-dimensional temperature are positive in case of absence of the thermal stratification parameter $(S=0)$, while $S=0.1$, $0.2,0.4,0.5$, the non-dimensional temperature are negative within the boundary layer, because at these values of $S$, the difference between the surface of the plate and ambient temperatures at $X=1$ is zero. It shows that greater cooling of the surface with an increase in $S$ results in a decrease in the temperature. It is due to the fact that an increase in the values of the thermal stratification parameter has the propensity to increase the thermal buoyancy effect. It takes more time to reach the steady state when $\mathrm{S}$ is diminutive.

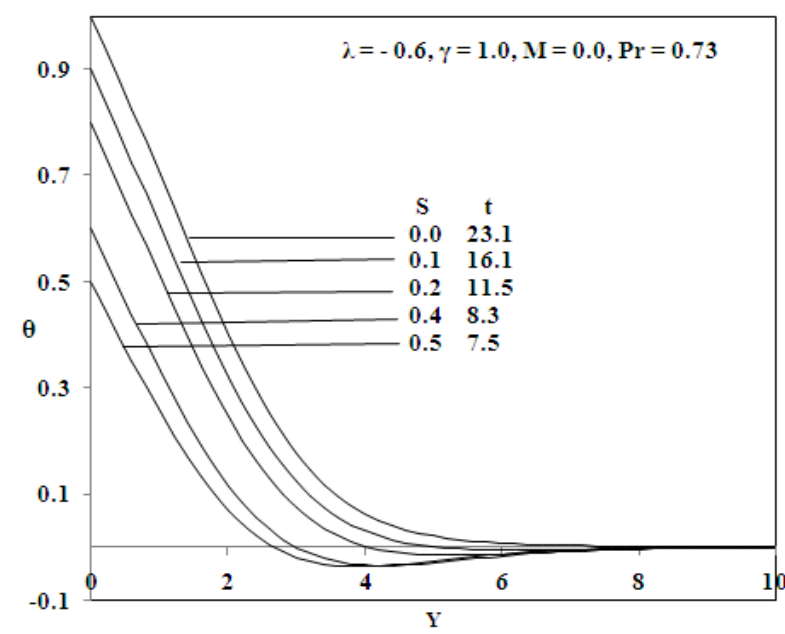

Fig 6: Steady state temperature profiles for different values of $S$

Figure 7 shows that, increasing the values of $\lambda, \gamma$ and $M$ produces increasing temperature gradients near the wall. Moreover, extreme cases of large values of the same parameters and some fixed values of $S$ show, far steeper temperature profiles. Figure 8 represents that the temperature decreases as the stratified parameter increases with increasing time for water.

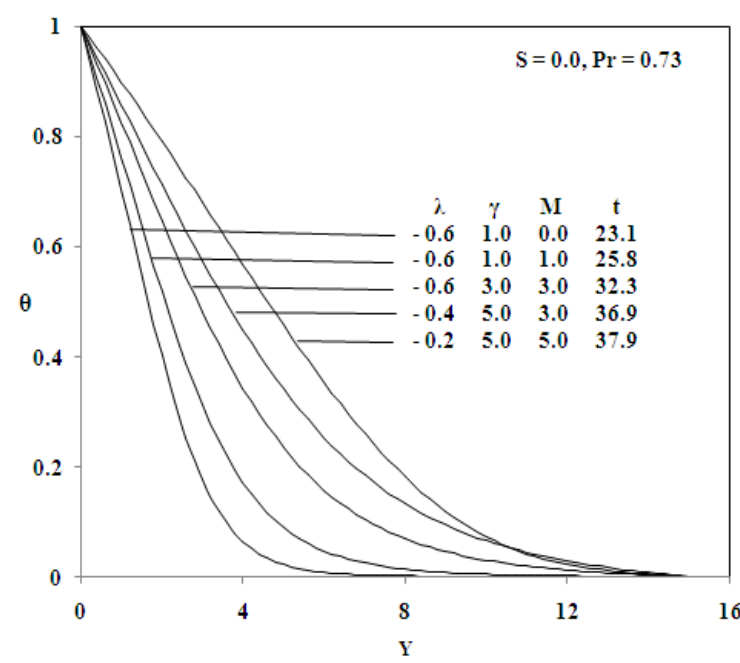

Fig 7: Steady state temperature profiles for different values of $\lambda, \gamma$ and $M$ 


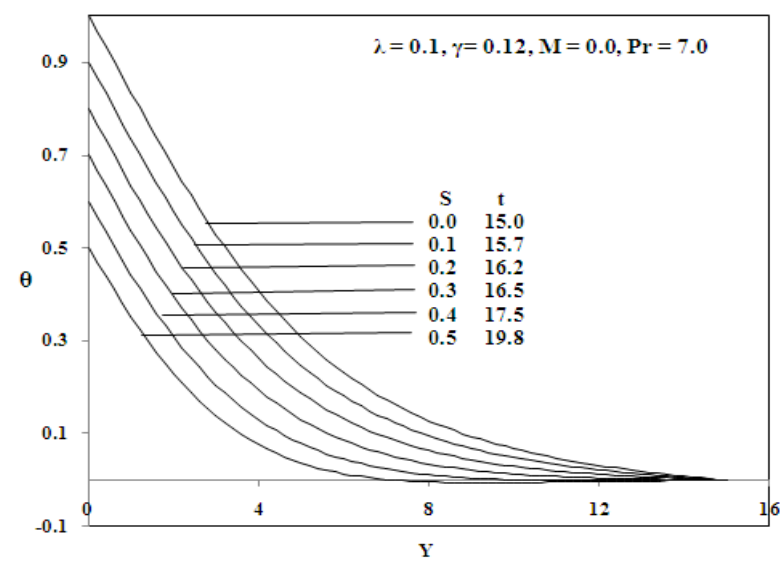

Fig 8: Steady state temperature profiles for different values of $S$

Knowing the velocity and temperature field, it is standard to study the physical quantities of fundamental interest of the skinfriction, the rate of heat transfer in steady state conditions. The dimensionless local and additionally average values of the skinfriction and Nusselt number can be expressed as

$$
\begin{aligned}
& C_{f}=\exp (-\lambda \theta)\left(\frac{\partial U}{\partial Y}\right)_{Y=0} \\
& \overline{C_{f}}=\exp (-\lambda \theta) \int_{0}^{1}\left(\frac{\partial U}{\partial Y}\right)_{Y=0} d X \\
& N u_{X}=-(1+\gamma \theta) G r^{1 / 4}\left(\frac{\partial \theta}{\partial Y}\right)_{Y=0} \\
& \overline{N u}=-(1+\gamma \theta) G r^{1 / 4} \int_{0}^{1}\left(\frac{\partial \theta}{\partial Y}\right)_{Y=0} d X
\end{aligned}
$$

The derivations involved in equations (11) and (12) are evaluated using five point formula and integrals are evaluated using Newton cotes formula numerically and from these equations, the local and average skin frictions are calculated. Figure 9 demonstrates that the local skin friction for different values of S and Prandtl numbers of air and water. It is observed from the figure that local skin friction decrease as increase in the stratification parameter $\mathrm{S}$ for both air and water. This is because, the velocity of the fluid decreases by increasing the stratification parameter as depicted in Figure 2. Therefore, there is a reduction in the shear stress along the wall and hence a decrease in the skin friction. It is additionally observed that an increase in Prandtl value, local skin friction is found to increase. The local and average heat transfer rates are calculated from the equations (13) and (14) respectively. Figures 10 and 11 represent the dimensionless steady state local heat transfer rate for air and water. From Figure 10, it is observed that the local heat transfer rate decreases as increasing the values of $\lambda, \gamma$ and M. Similarly, Figure 11 depicts the local heat transfer rate decreases as the thermal stratification decreases. Figure 12 shows that the average skin friction for water. It is observed from the figure that the average skin friction decreases for decreasing thermal conductivity parameter $\gamma$ but increasing magnetic parameter $\mathrm{M}$. This is because, the velocity of the fluid decreases by increasing the stratification parameter as shown from Figure 4. Figure 13 represents the average heat transfer rate for air and water. It decreases sharply at small values of time t, being unaffected by the stratification parameter $S$, but at large values of $t$, it is independent of time. When time increases, the average heat transfer increases and after some fluctuations, and reaches the asymptotic steady state. It is to be noted that the average heat transfer rate increases as the stratification parameter $\mathrm{S}$ increases for both air and water. It is also observed that a decrease in the Prandtl value, leads to an increase in the average heat transfer rate.

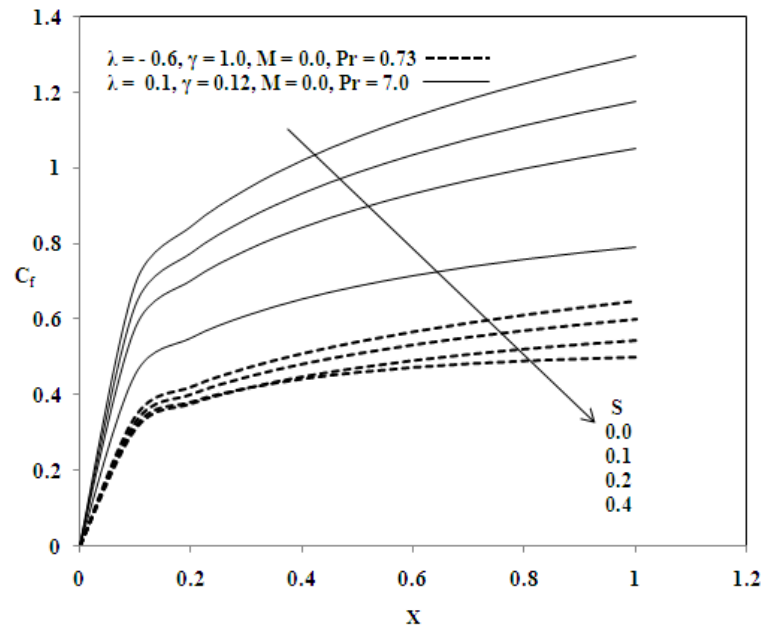

Fig 9: Local Skin friction

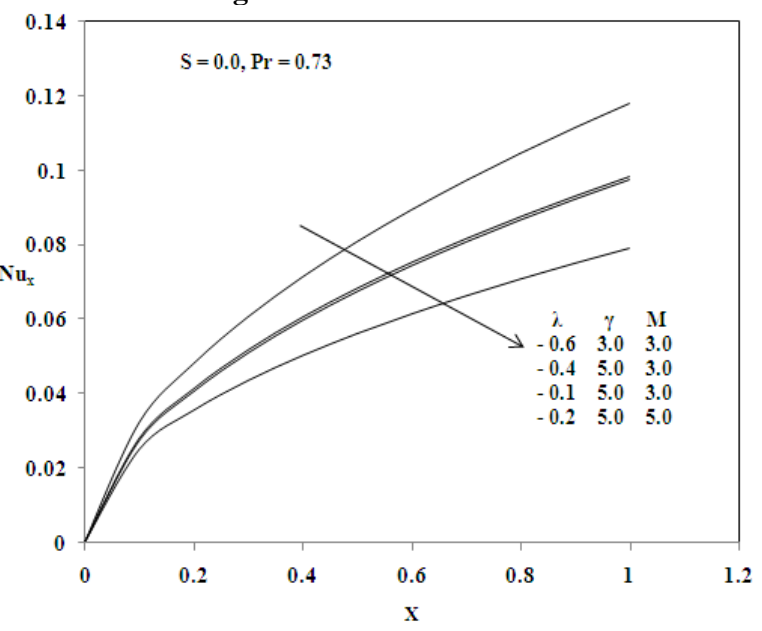

Fig 10: Local Nusselt number for different values of

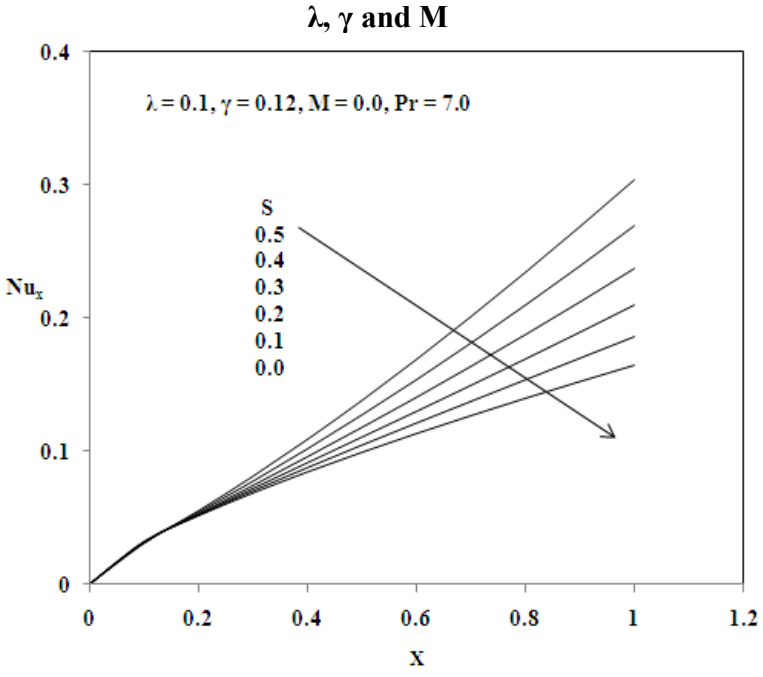

Fig 11: Local Nusselt number for different values of $S$ 


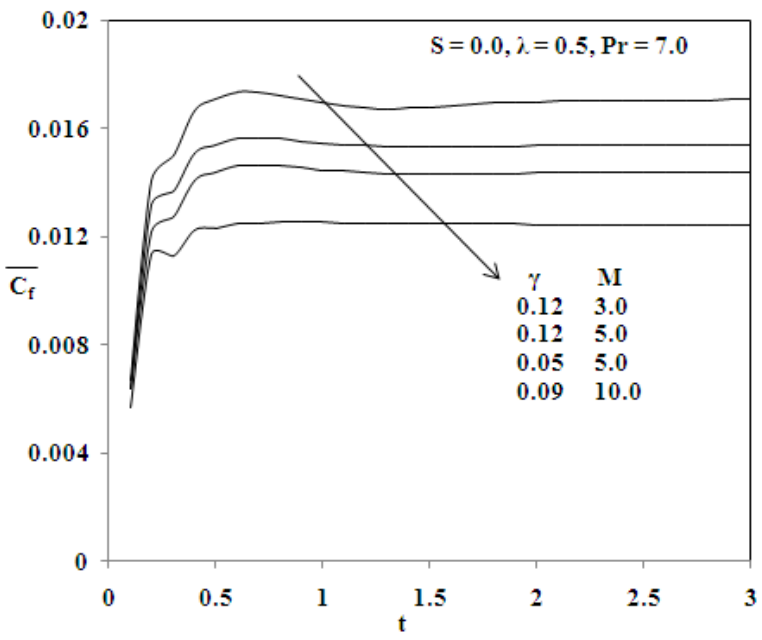

Fig 12: Average Skin friction for different values of $\gamma$ and $M$

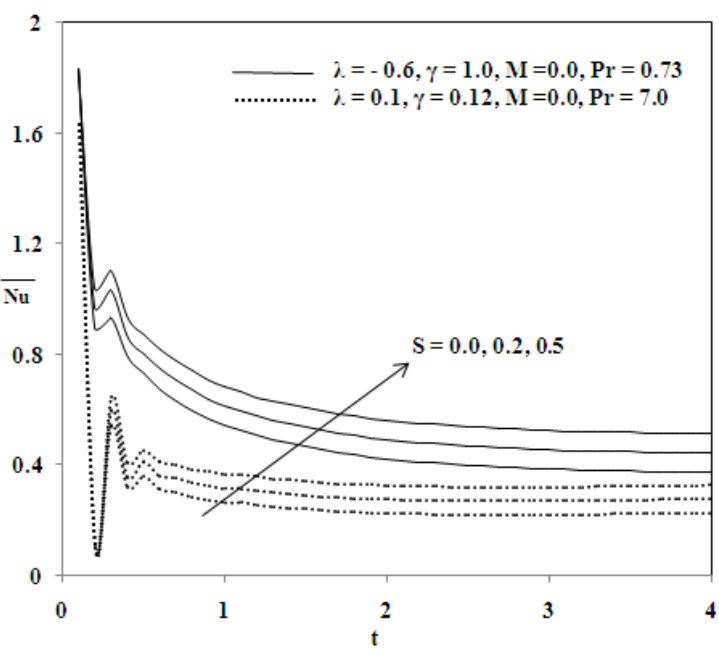

Fig 13: Average Nusselt number for different values of $S$ and $P r$

\section{CONCLUSIONS}

In this study, the problem of unsteady MHD natural convection flow over a semi-infinite vertical plate, in a thermally stratified media with variable viscosity and thermal conductivity is considered, and it is solved by the finitedifference method of the Crank-Nicolson type. This study concludes with the following results:

The velocity increases with a decrease in the stratified parameter for both air and water while the velocity increases with a decrease in the magnetic parameter $\mathrm{M}$, and increase in the viscosity parameter $\lambda$ for water. The temperature decreases on increasing $\mathrm{S}$ for both air and water. But the temperature decreases on decreasing $\lambda, \gamma$ and $\mathrm{M}$ for air. Local skin friction decreases with an increase in the stratification parameter $\mathrm{S}$ for both air and water. The local heat transfer rate decreases on increasing the values of $\lambda, \gamma$ and $\mathrm{M}$ for air, but decreases as the value of stratification parameter $\mathrm{S}$ decreases for water. The average skin friction decreases on decreasing $\gamma$ but on increasing $\mathrm{M}$ for water. The average heat transfer rate increases as the stratification parameter $\mathrm{S}$ increases for both air and water.

The present study may be established in various scientific aspects.

\section{REFERENCES}

[1] Soundalgekar, V. M. 1977. Free convection effects on the Stokes problem for an infinite vertical plate, Trans. ASME J. Heat transfer, Vol. 99, pp. 499-50.

[2] Hellums, J. D. and Churchill, S. W. 1962. Transient and steady state and natural convection", Numerical solution: Part 1, The isothermal vertical plate, American Institute of Chemical Engineers Journal, Vol. 8, pp. 690-692.

[3] Soundalgekar, V. M. and Ganesan, P. 1981. Finite difference analysis of transient free convection with mass transfer of an isothermal vertical flat plate, International Journal of Engineering Science, Vol. 19, pp. 757-770.

[4] Soundalgekar, V. M. and Mohammed Abdulla Ali. 1986. Free convection effects on MHD flow past an impulsively started infinite vertical isothermal plate, Reg. J. Energy Heat and Mass Transfer, Vol. 8(2), pp. 119125.

[5] Seddeek, M. A. 2002. The effect of variable viscosity on a MHD free convection flow past a semi-infinite flat plate with an aligned magnetic field in the case of unsteady flow, International Journal of Heat Mass Transfer, Vol. 45, pp. 931-935.

[6] Elbashbeshy, E. M. A. 2000. Free convection flow with variable viscosity and thermal diffusivity along a vertical plate in the presence of magnetic field, International Journal of Engineering Science, Vol. 38, pp. 207-213.

[7] Deka, R. K. and Neog, B. C. 2009. Unsteady natural convection flow past an accelerated vertical plate in a thermally stratified fluid, Theoretical Applied Mechanics, 36 (4), pp. 261-274.

[8] Kulkarni, A. K.., Jacobs, H. R., and Hwang, J. J. 1986. Similarity solutions for natural convection flow over an isothermal vertical wall immersed in thermally stratified medium, International Journal of Heat and Mass transfer, 130 (4), pp. 691-698.

[9] Gurminder Singh, R., Sharma, P. R., and Chamkha, A. J. 2010. Effect of thermally stratified ambient fluid on MHD convective along a moving non-isothermal vertical plate, International Journal of Physical Sciences, 5(3), pp. 208-215.

[10] Takhar, H. S., Chamkha, A. J., and Nath, G. 2001. Natural convection flow from a continuously moving vertical surface immersed in thermally stratified medium, Heat Mass Transfer, Vol. 38, pp. 17-24.

[11] Saha, S. C., and Hossain, M. A. 2004. Natural convection flow with combined buoyancy effects due to thermal and mass diffusion in thermally stratified media, Nonlinear Analysis: Modeling and Control, 9 (1), pp. 89 102.

[12] Hazarika, G. C., and Sarma, U. 2011. Effects of variable viscosity and thermal conductivity on heat and mass transfer flow along a vertical plate in the presence of a magnetic field, Lat. Am. J. Phys. Educ. 5 (1), pp. 100106. 
[13] Mahanti, N. C., and Gaur, P. 2009. The effects of varying viscosity and thermal conductivity on steady free convective flow and heat transfer along an isothermal vertical plate in the presence of heat sink, Journal of Applied Fluid Mechanics, 2 (1), pp. 23-28.

[14] Gnaneswara Reddy, M., and Bhaskar Reddy, N. 2009. Unsteady MHD convective heat and mass Transfer past a semi-infinite vertical porous plate with variable viscosity and thermal conductivity, International Journal of Applied Mathematics and Computation, 1(2), pp. 104117.

[15] Thakur, P. M., and Hazarika, G. C. 2015. Studied the effects of variable viscosity and thermal conductivity on unsteady free convective heat and mass transfer MHD flow of micropolar fluid with constant heat flux through a porous medium, International Journal of Computer Applications, Vol. 110 (8), pp. 20-30.

[16] Elbashbeshy, E. M. A. and Ibrahim, F. N. 1993. Steady free convection flow with variable viscosity and thermal diffusivity along a vertical plate, Journal of Physics, D Applied Physics, 26 (12), pp. 2137-2143.

[17] Elbarbary, E. M. E. and Elgazery, N. S. 2004. Chebyshev finite difference method for the effects of variable viscosity and variable thermal conductivity on heat transfer from moving surfaces with radiation, International Journal of Thermal Science, Vol. 43, pp. 889-899.

[18] Carnahan, B., Luther, H. A., and Wilkes, J. O. 1969. Applied Numerical Methods, John Wiley and Sons, New York. 